\title{
Relación entre autoestima y capacidad de agencia de autocuidado del paciente con tuberculosis pulmonar en el Hospital Huaycán, Lima, 2015
}

\author{
Relationship between self-esteem and self-care agency capacity of patients with pulmonary \\ tuberculosis in hospital Huaycán. Lima, 2015
}

Rocío Suarez Rodríguez¹, Irene Zapata Silva²

\begin{abstract}
RESUMEN
Objetivo: El objetivo del estudio fue determinar la relación entre la autoestima y la capacidad de agencia de autocuidado del paciente con tuberculosos pulmonar del Hospital de Baja Complejidad "Huaycán. Material y métodos: Investigación básica, de campo, descriptiva de diseño correlacional y transversal; la muestra estuvo conformada por 68 pacientes con tuberculosis pulmonar. Como instrumentos de recolección de datos se utilizaron la escala de autoestima de Rosemberg y la escala de apreciación de la agencia de autocuidado ASA (Appraisal of self-care Agency Scale) de Isenberg y Evers. Resultados: Los resultados muestran que existe relación significativa entre la variables autoestima y agencia de autocuidado $(\mathrm{r}=0.261, \mathrm{P}=0.031)$, en cuanto a la relación entre la autoestima y las dimensiones de la agencia de autocuidado, se encontró relación directa y significativa con la dimensión interacción social $(\mathrm{r}=0.299, \mathrm{P}=0.013)$; sin embargo, no se encontró relación significativa con las dimensiones de bienestar personal, actividad y reposo, consumo suficiente de alimentos, y promoción del funcionamiento y desarrollo personal. Conclusiones: Se concluye que existe relación significativa entre el nivel de autoestima y la interacción social del paciente con tuberculosis pulmonar en el Hospital de Baja Complejidad "Huaycán”.
\end{abstract}

Palabras clave: Autoestima, capacidad de agencia de autocuidado, tuberculosis.

\begin{abstract}
Objective: The objective of this study was to determine the relationship between self-esteem and self-care agency capacity of patients with pulmonary tuberculosis from the Low Complexity Hospital "Huaycán". Methods: Basic research, local research, non experimental, transversal, descriptive and correlational design, the sample was 68 patients with pulmonary tuberculosis. The data collection instruments were the Rosenberg Self-Esteem Scale and the Scale of assessment of self-care agency ASA (Appraisal of Self-Care Agency Scale) of Isenberg and Evers. Results: The results showed that there is significant correlation $(\mathrm{r}=0.261, \mathrm{P}=0.031)$ as to the relationship between self-esteem and the dimensions of self-care agency, direct and significant relation was found social interaction dimension $(\mathrm{r}=0.299, \mathrm{P}=0.013)$; however, no significant relative to the size of personal, activity and rest, sufficient food consumption, and promotion of personal development and operation was found. Conclusions: It is concluded that there is significant relationship between self-esteem and social interaction of patients with pulmonary tuberculosis in Low Complexity Hospital "Huaycán".
\end{abstract}

Keywords: Capacity of self-care agency, life quality in health, elderly

\footnotetext{
${ }^{1}$ Coordinadora de Especialidades en Enfermería, Unidad de Posgrado de Ciencias de la Salud, Universidad Peruana Unión, Lima, Perú. ${ }^{2}$ Magister en Enfermería, Miembro del Equipo de Gestión de la Calidad y Control Interno en el Hospital Nacional Guillermo Almenara, Lima Perú.
} 


\section{INTRODUCCIÓN}

Enfermería es una disciplina de la salud que orienta sus acciones al logro del mejoramiento continuo de la calidad de vida de los individuos, familia y comunidad (Mendes, 2001).

Desde esta perspectiva, el profesional de Enfermería no puede ser ajeno a la problemática de salud que afecta a la sociedad, tomando acciones de cuidado pertinentes.

La OMS (2015) reporta que, a nivel mundial, la tuberculosis es la segunda causa de mortalidad, después del SIDA, el mayor número de casos ocurrió en Asia Sudoriental y en regiones del Pacífico Occidental y África, en Latinoamérica cada año se diagnostican 230 mil casos nuevos de tuberculosis y más de 53 mil personas mueren a causa de esta enfermedad. A nivel nacional la prevalencia de tuberculosis es de 275 por cada 100.000 personas y afecta a 32 mil personas cada año, de las cuales el $60 \%$ se encuentra en Lima Metropolitana, Callao, Loreto y La Libertad (MINSA, 2013).

En este contexto, es importante destacar el rol de la enfermera en la promoción del autocuidado para lograr un compromiso y participación por parte del paciente y su familia en el tratamiento, siendo necesario conductas permanentes de autocuidado que faciliten el conocimiento, empoderamiento del individuo y el desarrollo de la capacidad en la toma de decisiones sobre su salud

\section{MATERIAL Y MÉTODOS}

El estudio fue descriptivo, de diseño correlacional y transversal, porque se orienta a describir la capacidad de agencia de autocuidado y la autoestima, además de determinar la relación entre ambas (Hernández, R., Fernández, C. y Baptista, M. 2010). La población estuvo conformada por todos los pacientes con TBC que pertenecen al Hospital de Baja Complejidad “Huaycán”, con un total de 113 pacientes; la muestra fue de 68 pacientes, determinada mediante muestreo no probabilístico por conveniencia para el estudio.

Instrumentos: se utilizó la escala de Autoestima de Rosemberg, que consta de 10 ítems enunciados de forma positiva y negativa; la escala de valoración final varía entre 10 y 40 puntos, dando como resultado 3 niveles de autoestima: alto, medio y bajo. La consistencia interna para el instrumento validado en español es de $\alpha=0.87$, que es muy similar a los valores de 0.77 y 0.88 encontrado en otros estudios. La escala de apreciación de la agencia de autocuidado ASA (Appraisal of selfcare Agency Scale) de Isenberg y Evers (1993), validada en español por Gallegos (1998), que consta de 24 ítemes con un formato de respuesta con 5 alternativas estilo Likert, donde el número uno (nunca) significa el valor más bajo de capacidad de agencia de autocuidado y el cinco (siempre) el valor más alto. Los puntajes varían de 24 a 120 puntos, teniendo 3 niveles: alto, medio y bajo. Los valores de fiabilidad para el instrumento es de $\alpha=0.6891$ con un valor estandarizado de 0.7440 .

Los datos obtenidos se procesaron con el programa estadístico SPSS versión 20. Se aplicó la prueba Kolmogorov-Smirnov (K-S) para determinar si las puntuaciones de cada variable tienen una distribución normal; los valores obtenidos para la variable Autoestima fue $\mathrm{K} \_S=0.79$, $\mathrm{p}=0.55$ y para la variable Autocuidado K-S=0.73, $\mathrm{p}=0.066$, indicando que las puntuaciones de ambas variables se ajustaron a una distribución normal, haciendo posible el estudio de relación de las dos variables a través de la prueba estadística de correlación de Pearson. El nivel de significancia utilizado para desarrollar el análisis estadístico fue de $\alpha=5 \%$.

\section{RESULTADOS}

Tabla 1

Distribución de frecuencias de la autoestima y la capacidad de agencia de autocuidado en pacientes con TBC pulmonar en el Hospital de Baja Complejidad "Huaycán”.

\begin{tabular}{|c|c|c|c|c|c|}
\hline \multirow[b]{3}{*}{ Autoestima } & \multicolumn{2}{|c|}{ Bajo } & \multicolumn{2}{|c|}{ Medio } & Alto \\
\hline & & $\%$ & & $\%$ & $\mathrm{n} \%$ \\
\hline & 17 & 25.0 & 38 & 55.9 & 1319.1 \\
\hline Agencia de autocuidado & 18 & 26.5 & 17 & 25.0 & 3348.5 \\
\hline Interacción social & 13 & 19.1 & 44 & 64.7 & 1116.2 \\
\hline Bienestar personal & 15 & 22.1 & 40 & 58.8 & 1319.1 \\
\hline Actividad y reposo & 15 & 22.1 & 36 & 52.9 & 1725.0 \\
\hline Consumo de alimentos & 6 & 8.8 & 29 & 42.6 & 3348.5 \\
\hline Promoción del funcionamiento y desarrollo personal & 14 & 20.6 & 43 & 63.2 & 1116.2 \\
\hline
\end{tabular}


En la tabla 1, se observa que el $55.9 \%$ de la muestra tiene un nivel de autoestima medio, seguido del $25.0 \%$ con nivel bajo y $19.1 \%$ con nivel alto; el $48.5 \%$ presenta un nivel alto de capacidad de agencia de autocuidado, el $26.5 \%$ y $25.0 \%$ presenta nivel bajo y medio respectivamente. Respecto a las dimensiones de la capacidad de agencia de autocuidado, los porcentajes más altos corresponden al nivel medio en las dimensiones de interacción social, promoción del funcionamiento y desarrollo personal, bienestar personal y actividad y reposo con el 64.7\%, $63.2 \%, 58.8 \%$ y $52.9 \%$ respectivamente; seguidas del nivel alto en las dimensiones de consumo de alimentos, y actividad y reposo con el $48.5 \%$ y $25 \%$ respectivamente.

\section{Tabla 2}

Relación entre la autoestima y la capacidad de agencia de autocuidado según dimensiones en pacientes con tuberculosis pulmonar del Hospital de Baja Complejidad “Huaycán”. Octubre 2014.

\begin{tabular}{lccc}
\hline \multicolumn{1}{c}{ Agencia de autocuidado /dimensiones } & \multicolumn{3}{c}{ Autoestima } \\
\cline { 2 - 4 } & $\mathrm{n}$ & $\mathrm{r}$ & $\mathrm{p}$ \\
\hline Interacción social & 68 & 0.299 & 0.013 \\
Bienestar personal & 68 & 0.193 & 0.115 \\
Actividad y reposo & 68 & 0.229 & 0.060 \\
Consumo suficiente de alimentos & 68 & 0.148 & 0.229 \\
Promoción del funcionamiento y desarrollo personal & 68 & 0.216 & 0.077 \\
\hline
\end{tabular}

La tabla 2 muestra una relación significativa entre la variable autoestima y capacidad de agencia de autocuidado ( $r=0.261, \mathrm{p}=0.031$ ). En cuanto a la relación entre la autoestima y las dimensiones de la capacidad de agencia de autocuidado, se observa una relación directa y significativa con la dimensión interacción social ( $\mathrm{r}=0.299$, $\mathrm{p}=0.013)$; no hay relación significativa con las dimensiones bienestar personal, actividad y reposo, consumo suficiente de alimentos, y promoción del funcionamiento y desarrollo personal.

\section{DISCUSIÓN}

Los resultados del presente estudio muestran que más de la mitad de los pacientes con tuberculosis pulmonar tienen un nivel medio de autoestima (55,99\%), la cuarta parte tiene un nivel bajo (25\%), y un mínimo porcentaje tiene un nivel alto $(19,1 \%)$. Estos resultados evidencian el modo cómo la enfermedad vulnera la valoración de sí mismo por parte del paciente y confirma el efecto desfavorable potencial advertido por McKay y Fanning (1999), quien señala la importancia de la autoestima en las expectativas y motivación de la persona para mantener su equilibrio psíquico.

El nivel bajo de autoestima encontrado puede deberse a los efectos que la enfermedad ocasiona directamente en la valoración que el paciente tiene de sí mismo, quien experimenta sentimientos de poca confianza y escaso valor para hacer frente a los desafíos de su recuperación y a la lucha por sus derechos ciudadanos (Morrison,1996); lo que demanda por parte de la enfermera y los miembros del equipo de salud, una mayor aceptación, actitud comprensiva y empática, que les permita alcanzar el sosiego y la estabilidad para actuar superando los estados de ánimo negativos y poco entusiastas (Wilber, 1995).

El conocimiento de la autoestima de los pacientes afectados de tuberculosis pulmonar es fundamental para la toma de decisiones e intervención de enfermería en la labor asistencial, educativa y de gestión de acciones teniendo en cuenta las características individuales y demandas sociales de los sujetos de cuidado (Uribe, 1999). Así mismo, Tobón (2004) destaca de manera contundente la importancia de considerar la valoración de la propia estima para fortalecer los factores personales que determinan la manera de cuidarse y proteger la salud.

En cuanto a la capacidad de agencia de autocuidado, se encontró que casi la mitad de la muestra presenta un nivel alto a pesar de tener un nivel medio de autoestima, indicando que conservan la capacidad para cuidar de sí mismos aún con las limitaciones que le genera la enfermedad, explicable por la edad de los pacientes de la muestra, pues el $52.9 \%$ tenía entre 21 y 40 años correspondiendo a la etapa del adulto joven, caracterizado por tomar decisiones importantes respecto a su salud, carrera y relaciones personales (Papalia, 2005).

Asimismo, la mitad de los pacientes son de estado civil de conviviente o casado, reflejando que cuentan con una pareja que constituye el apoyo social más cercano para incentivarlos en la práctica del autocuidado. Resultados similares corresponden al estudio realizado por Leiva, Acosta, Berrocal, 
Carrillo, Castro, y Watson (2012) en personas adultas con artritis reumatoide tipo 1 , quienes encontraron una alta capacidad de agencia de autocuidado en el 84.6\% de estos pacientes; del mismo modo, Herrera, A., Andrade, Y., Hernández, S., Manrique, J., Faria, K. y Machado, M. (2012) en el estudio realizado en pacientes con diabetes mellitus tipo 2, encontraron muy buena capacidad de agencia de autocuidado en el $76 \%$ de la muestra.

El análisis de los resultados descritos, representa un insumo relevante para la promoción del autocuidado por parte de los enfermeros del Hospital de Baja Complejidad "Huaycán”, teniendo como meta aumentar el porcentaje de pacientes con mayor capacidad de agencia de autocuidado independientemente de su estado de salud, asumiendo que les ayudaría a recuperarse en menor tiempo y reintegrarse a la sociedad. Por tanto, es necesario comprender que el autocuidado no es solamente un acto intelectual y teórico, sino ante todo práctico que necesita ser desarrollado por el paciente con el apoyo del personal de enfermería integrando esfuerzos y estrategias en el acto de cuidar, entendido como la interacción humana, de carácter interpersonal, intencional y único que sucede en un contexto sociocultural, con un fin determinado y que refuerza la dignidad humana (Watson y Leininger, citadas por Pinzón, 2000).

En cuanto a la relación entre la autoestima y la capacidad de agencia de autocuidado, se encontró que existe una relación significativa positiva, lo que indica que si se mejora la autoestima de los pacientes, también mejorará su capacidad de agencia de autocuidado, corroborando la posición del MINSA (2008) quien afirma que la autoestima es fundamental en el autocuidado; es decir, el autocuidado es en gran parte el resultado del quererse (Tobón, 2003); si la persona tiene una visión buena de sí misma, puede desarrollar fácil y benéficamente una cultura de autocuidado en todas sus dimensiones (Dirección Nacional de Prevención de Riesgos Profesionales. Plan Integral de Capacitación y Asistencia Técnica).

Al evaluar la relación entre el nivel de autoestima y las dimensiones de la capacidad de agencia de autocuidado, sólo se encontró una relación significativa con la dimensión de interacción social debido a que esta dimensión proporciona seguridad, confianza y capacidad de reflexión en uno mismo y con relación a otras personas (Cárdenas, 2009).

Al respecto, García (2005) menciona que si la interacción social en pareja o en grupo es positiva, genera mayores fuentes de autoestima y bienestar personal, así mismo afirma que la habilidad social es importante en la prevención o control de problemas de salud. Sin embargo, al considerar el apoyo social, es necesario tener en cuenta que no sólo basta la calidad de relación que se tenga con familiares y amigos, el nivel de participación en instituciones sociales o el número y calidad de servicios que brinda la sociedad, sino también, cómo el paciente evalúa y percibe el apoyo recibido. Por tanto, para que esta percepción sea positiva, es necesario que el apoyo se realice cuando se necesite, como se necesita y por quien se necesita (Zaldívar, 1999; citado por Hernández, 2009).

A pesar de la importancia que tiene la interacción social, Manosalva, Espinoza y Mateus (2008), mencionan que generalmente la población enferma no cuenta con redes de apoyo suficientes para satisfacer las necesidades de autocuidado cotidiano y las demandas incrementadas por la enfermedad, lo que repercute en su nivel de autoestima, interfiriendo en el desarrollo de su capacidad de agencia de autocuidado. Esta realidad, se evidencia en los pacientes con tuberculosis pulmonar donde la familia, que es el apoyo social más cercano, muchas veces se cansa, los pacientes tienen que abandonar el trabajo y son olvidados por sus amigos por temor al contagio, situación que afecta su aspecto emocional y social.

No se encontró relación significativa entre la autoestima y las demás dimensiones de la capacidad de agencia de autocuidado, probablemente porque a pesar de contar con un buen porcentaje de jóvenes que tienen cierto grado de responsabilidad en la toma de decisiones respecto al cuidado de su salud, también intervinieron adolescentes, adultos intermedios y adultos mayores, quienes tienen sus propias características.

El haber encontrado casi la mitad de la muestra con un nivel alto en la dimensión de consumo de alimentos, es considerado como un resultado relevante sobre todo en los pacientes con tuberculosis pulmonar, pues ésta enfermedad hace que el organismo gaste más energía diaria necesitando una alimentación equilibrada que mejore su estado nutricional, la respuesta inmunológica, el transporte de fármacos y la reparación tisular. La nutrición del paciente con tuberculosis debe ocupar un lugar prioritario ya que se ha demostrado que muchos fármacos antituberculosos actúan provocando trastornos gastrointestinales que alteran la absorción de nutrientes, además de ocasionar nauseas, vómitos y alteraciones de los sentidos del gusto y del olfato, provocando la disminución en el consumo de alimentos y por ende deterioro del estado nutricional. Dentro de este contexto, el profesional de enfermería 
como miembro importante del equipo de salud, tiene dentro de sus funciones el desarrollo de actividades de promoción y prevención que permitan a los pacientes orientar sus actividades de autocuidado hacia una alimentación saludable (Oria, 2006 y Ccoica, 2013).

Constatar que más del $50 \%$ de los pacientes presentó un nivel medio de capacidad de agencia de autocuidado en las dimensiones de promoción del funcionamiento y desarrollo personal, bienestar personal, y actividad y reposo, revela que las estrategias desplegadas por el personal de salud en este grupo de pacientes muestra avances en el aprendizaje y desarrollo de capacidades del autocuidado básico de la persona; consecuencia favorable que amplía las fronteras de intervención de la enfermera y la impulsa a continuar su labor para establecer cada vez más, una relación terapéutica legítima y apropiada con los pacientes que padecen tuberculosis pulmonar (Cutcliffe, McKenna y Hykäs, 2011).

Finalmente, teniendo en cuenta que más de la mitad de la muestra, presenta un nivel medio de autoestima, es un reto para el personal de salud, en especial para el profesional de enfermería, formular estrategias orientadas a alcanzar un nivel alto de autoestima en los pacientes con tuberculosis pulmonar, considerando el riesgo que el nivel de autoestima disminuya afectando su capacidad de agencia de autocuidado, debido a la relación directa y significativa demostrada en el presente estudio, siendo primordial involucrar a la familia quien constituye el pilar terapéutico y apoyo social insustituible para el paciente.

\section{Declaración de financiamiento y de conflicto de intereses:}

El estudio fue financiado por los autores, quienes declaran no tener algún tipo de conflicto de interés en la investigación realizada.

\section{Correspondencia:}

\section{Rocío Suárez Rodríguez}

Universidad Peruana Unión, Carretera Central Km.19.5 Ñaña

e-mail: rociojaeli@hotmail.com

\section{REFERENCIAS BIBLIOGRÁFICAS}

Cárdenas, N. \& Buitrago, H (2009) Nivel de agencia de autocuidado de la salud en el trabajo, en un grupo de trabajadores de la construcción de una Institución Educativa Privada de Nivel Superior. (Tesis para optar título de especialista en salud ocupacional). Universidad Javeriana. Colombia.

Ccoicca, F. (2013). Medidas de autocuidado que tienen los pacientes que asisten a la Estrategia Sanitaria Nacional-Programa del Control dela Tuberculosis del centro de salud Conde de la Vega Baja (Tesis de licenciatura). Universidad Nacional Mayor de San Marcos. Recuperado de: http://cybertesis.unmsm.edu. pe/bitstream/cybertesis/1043/1/ccoicca_af.pdf

Cutcliffe, J., McKenna, H., \& Hykas, K. (2010). Modelos de Enfermería: aplicación a la práctica. Bogotá. Manual Moderno.

Dirección Nacional de Prevención de Riesgos Profesionales. Plan Integral de Capacitación y Asistencia Técnica. Recuperado de: http://petrolerosasociados.com/ boletines/emergencias/brigadas.pdf

Fundichely, M., \& Zaldívar, I. (1999) Autoestima en el personal de enfermería. Revista Cubana de Enfermería. Recuperado de http://scielo.sld.cu/pdf/enf/v15n3/ enf09399.pdf
Gallegos E. (1998). Validez y confiabilidades de la versión en español de la escala: Valoración de las Capacidades de Autocuidado. México: Desarrollo Científico.

García P., Z. (2005). Conocimientos y prácticas que tienen los pacientes traqueostomizados sobre autocuidado en los consultorios de cabeza y cuello del Instituto Nacional de Enfermedades Neoplásicas. UNMSM. Perú.

Hernández E. (2009). Encuesta Nacional de Conocimiento, Actitud y Comportamiento de la Población ante la Tuberculosis. Cuba.

Hernández, R., Fernández, C. \& Baptista, M., (2010) Metodología de la Investigación. ( $5^{\mathrm{a}}$ ed.) México: Mc Graw Hill.

Herrera, A., Andrade, Y., Hernández, S., Manrique, J., Faria, K. \& Machado, M. (2012) Personas con diabetes mellitus tipo 2 y su capacidad de agencia de autocuidado, Cartagena. Universidad de Cartagena. Recuperado de http://www.scielo.org.co/pdf/aven/v30n2/v30n2a03.pdf

Leiva, V.; Acosta, P.; Berrocal, Y.; Carrillo, E.; Castro, M. \& Watson, Y. (2012) Capacidad de agencia de autocuidado en personas adultas con artritis reumatoide. Rev. Enfermería Actual en Costa Rica. Disponible http:// www.revenf.ucr.ac.cr/artritis.pdf/ 
Relación entre autoestima y capacidad de agencia de autocuidado del paciente con tuberculosis pulmonar en el Hospital Huaycán, Lima, 2015

Manosalva, M, Espinosa, X. \& Mateus, J. (2008). Capacidad de agencia de autocuidado de las personas portadoras de VIH/SIDA en una fundación de Bogotá. Noviembre. Pontificia Universidad Javeriana. Colombia.

Mendes, R. \& Velásquez, S. (2001). Teoría General del Autocuidado. Santa Fe de Bogotá Editorial UNIBIBLOS

MINSA. (2008). Plan de Control de infecciones intrahospitalarias por tuberculosis en Hospital de Baja Complejidad Huaycán.

MINSA. (2013). Boletín epidemiológico. 6 (3). Recuperado de http://www.rslc.gob.pe/Descargas/Epidemiologia/ Boletin/2013/Boletin-N-03- 2013.pdf

Mc Kay, Fanning, P. (1999). Autoestima. Evaluación y Mejora. Madrid: Ediciones Martínez Roca.

Morrison, J., (1996). Fundamentos de enfermería en salud mental. Madrid: Editorial Prentice Hall.

OMS, (2015) Tuberculosis. Nota descriptiva $\mathrm{N}^{0} 104$. Recuperado de http://www.who.int/mediacentre/ factsheets/fs104/es/

Oria, M. (2006). Conocimientos y prácticas que tienen los pacientes con tuberculosis sobre alimentación su relación con el estado nutricional en el centro de Salud
José Carlos Mariátegui, Lima. Universidad Nacional Mayor de San Marcos. Perú.

Papalia, D. (2005). Psicología del Desarrollo. México: Mc Graw Hill.

Pinzón, L., (2000). Hacia la renovación de la acreditación del programa de Enfermería de la Universidad de Caldas: anexos seleccionados, Marrizales: Programa de Enfermería. Julio. Recuperado: http://promocionsalud. ucaldas.edu.co/downloads/Revista\%208_5.pdf

Tobón, O. (2003). El autocuidado una habilidad para vivir. Revista Hacia la Promoción de la Salud [serie en línea]. Recuperado de http://promocionsalud.ucaldas. edu.co/downloads/Revista\%208_5.pdf

Tobón, O, (2004). El autocuidado, una habilidad para vivir. Recuperado de: http://promociónsalud,ucaldas,edu,co/ dowloads.

Uribe, T. (1999). El Autocuidado y su papel en la promoción de la Salud. Recuperado de: http://toneudea.edu.co/ revista/sep99/autocuidado.htm.

Wilber, K. (1995). El proyecto Atman. Barcelona: Editorial Kairós.

Recibido: 20/08/2015 Aceptado: 12/10/2015 